\title{
Investigation of Flow over the Airfoil NACA 0010-35 with Various Angle of Attack
}

\author{
Shiming Xiao ${ }^{1, a}$ and Zutai Chen ${ }^{1}$ \\ ${ }^{1}$ Department of Mechanical Engineering, Engineering Faculty, University of Portsmouth, The United Kingdom
}

\begin{abstract}
This report is using the finite element analysis method to investigate the performance of an airfoil (NACA 0100-35), which uses 2D computational fluid dynamics simulations based on ANSYS to find the lift and drag coefficients under different conditions. The boundary conditions are defined as follows: The density is $1 \mathrm{~kg} / \mathrm{m}^{3}$, gauge pressure is 0 , and airflow velocity is $50 \mathrm{~m} / \mathrm{s}$. Also the Reynolds number is considered during the simulation. As a result, the simulation shows the stalling points and efficiencies of the airfoil under different angles of attack. The simulation also plots the contours and stream lines in these conditions.
\end{abstract}

\section{Introduction}

Considering the important role the aircraft manufacturing has been played, selection of airfoils which is the section side of the airplane wings should be determined with certain conditions. One of the most popular softwares in this field is ANSYS, and it is used to obtain accurate results for simulating airfoils. The objective of this report is to investigate an airfoil (NACA 0010-35) with different angels of airflows based on ANSYS with 2D CFD (Computational Fluid Dynamics) simulation. The simulation is required to find the values of drag and lift coefficients in order to get stalling points and efficiencies.

\section{Parameters of the airfoil}

\subsection{Lift and Drag}

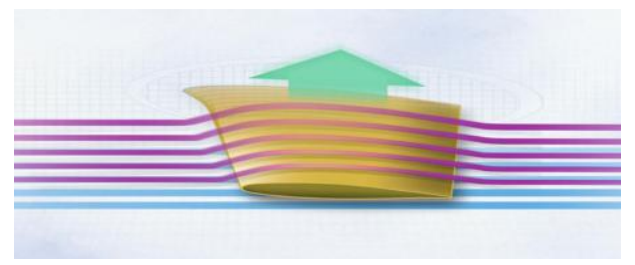

Figure 1. Airflow through airfoil

\footnotetext{
${ }^{\mathrm{a}}$ Corresponding author: flora901120@gmail.com
} 
According to Tom Benson's article[1], when an airfoil moves through airflow (as it is showed in figure 1), an aerodynamics force will be generated on it (as it is showed in figure 2 and figure 3 ). This aerodynamic force contains of the following:

$$
\begin{gathered}
L=\frac{1}{2} \rho v^{2} C_{l} A \\
D=\frac{1}{2} \rho v^{2} C_{d} A
\end{gathered}
$$

Where $D$ is the drag force, $L$ is the lift force, $\rho$ is the density of the fluid, $v$ is the speed of the object relative to the fluid, $A$ is the cross-sectional area, and $C_{d}$ is drag coefficient and $C_{L}$ is lift coefficient.

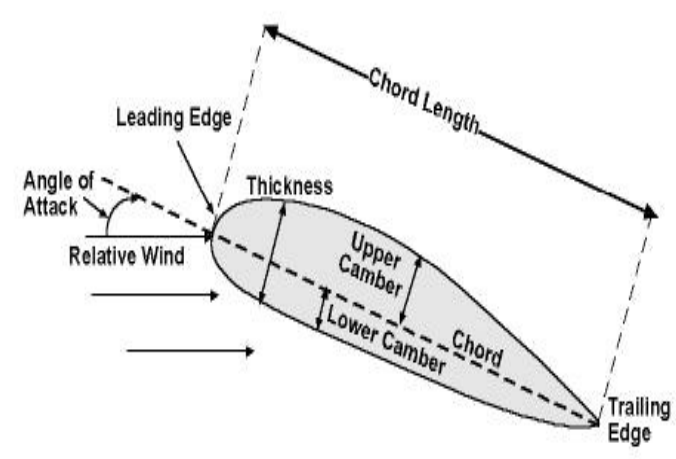

Figure 2. wing side view (airfoil)

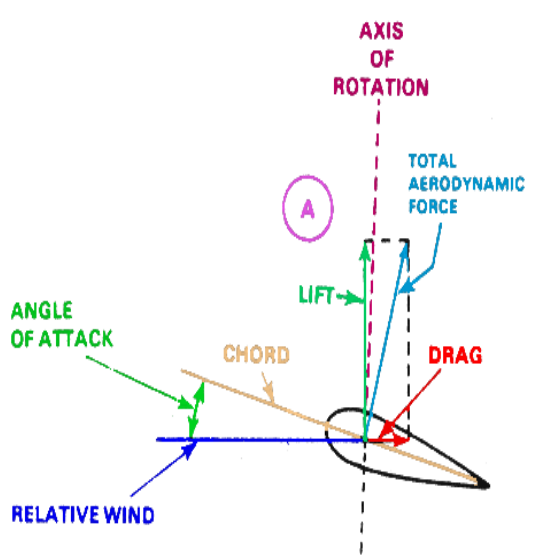

Figure 3. Lift and Drag forces Vs Angle of Attack

From the equation above, it can be illustrated that the lift force and drag force on any object are depend on the density of the fluid and the relative speed between the object and fluid proportionally.

\subsection{Lift and Drag}

The Lift Coefficient equation which could be rearranged from the Lift equation is as follow:

$$
C_{l}=\frac{2 L}{\rho v^{2} A}
$$

Where $\mathrm{L}$ is the lift, $\rho$ is the density of the fluid, $\mathrm{v}$ is the velocity of the fluid and A is the relevant surface area [2]. For the airfoil, the relevant surface area, which is related to the drag coefficient types, is a plane form area [3]. T. Benson [4] mentioned from NASA, the lift coefficient is usually determined experimentally, meanwhile it is a number which could describe all the complex dependencies of shape, inclination and some flow conditions on aircraft lift. In this report, $C_{l}$ is calculated from ANYSIS.

Also, the Drag Coefficient equation could be rearranged from the Drag equation as follow:

$$
C_{d}=\frac{2 D}{\rho v^{2} A}
$$

Where $\mathrm{D}$ is the drag force, which is by definition the force component in the direction of the flow velocity, $\rho$ is the mass density of the fluid, $v$ is the speed of the object relative to the fluid and $A$ is the reference area.

In this report, $\mathrm{Cl}$ and $\mathrm{Cd}$ could be calculated from the ANSYS system.

\subsection{Reynolds number}


The equation for Reynolds number is showed in following equation:

$$
R_{e}=\frac{\rho v D}{\mu}
$$

Where $\rho$ is the density for airflow $\left(1 \mathrm{~kg} / \mathrm{m}^{3}\right), \mathrm{v}$ is the velocity for airflow $(50 \mathrm{~m} / \mathrm{s}), \mathrm{D}$ is $1 \mathrm{~m}, \mu$ is the viscosity $(1.460 \mathrm{e}-5)$ for the air.

Patil et al.[5] investigated that the Lift and Drag forces at various angle of attack for Reynolds number from 10,000 to 800,000 by CFD analysis and concluded that the lift and drag forces increased with the Reynolds number increased. In this simulation, calculated by the above equation, $\mathrm{R}_{\mathrm{e}}$ is $3.42 \mathrm{x}$ $10^{6}$. So the flow is turbulent. This case only investigates the relationship between the angle of attack and its effects. The angle of attack, as it is showed in Figure 3, is the angle between the relative wind and the chord. As the leading edge which is front point of the airfoil turns up, angle of attack increases and connected to the increase of the lift force and drag force [3]. Investigations from former work from Sahin et al. [6] analysed the impact of stalling angle on lift and drag coefficient. Meanwhile, Bhat et al.[7] had also worked out the stalling angle of NACA0012 at a certain Reynolds number.

\section{Simulation}

\subsection{Choose an Airfoil}

Airfoil NACA 0010-35, as a symmetrical section, was chosen from the UIUC Airfoil Database(n.d.) to simulate. (showed in Figure 4 )

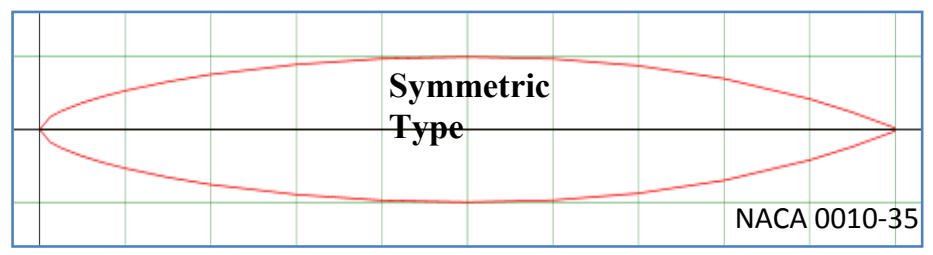

Figure 4. Geometry for NACA 0010-35

The data in Table 1 below were downloaded from the UIUC Airfoil Database(n.d.) and imported to the ANSYS workbench using Notepad.

Table 1. Data for NACA 0010-35 Airfoil

\begin{tabular}{|l|l|l|l|}
\hline Point & x-cord & y-cord & z-cord \\
\hline 1 & 0.00000 & 0.00000 & 0.00000 \\
\hline 2 & 0.0125 & 0.00878 & 0.00000 \\
\hline 3 & 0.0250 & 0.01267 & 0.00000 \\
\hline 4 & 0.0500 & 0.01844 & 0.00000 \\
\hline 5 & 0.0750 & 0.02289 & 0.00000 \\
\hline 6 & 0.1000 & 0.02667 & 0.00000 \\
\hline 7 & 0.1500 & 0.03289 & 0.00000 \\
\hline 8 & 0.2000 & 0.03789 & 0.00000 \\
\hline 9 & 0.3000 & 0.04478 & 0.00000 \\
\hline 10 & 0.4000 & 0.04878 & 0.00000 \\
\hline 11 & 0.5000 & 0.05000 & 0.00000 \\
\hline 12 & 0.6000 & 0.04867 & 0.00000 \\
\hline 13 & 0.7000 & 0.04389 & 0.00000 \\
\hline 14 & 0.8000 & 0.03500 & 0.00000 \\
\hline
\end{tabular}




\begin{tabular}{|l|l|l|l|}
\hline 15 & 0.9000 & 0.02100 & 0.00000 \\
\hline 16 & 0.9500 & 0.01178 & 0.00000 \\
\hline 17 & 1.0000 & 0.00100 & 0.00000 \\
\hline 18 & 1.0000 & -0.00100 & 0.00000 \\
\hline 19 & 0.9500 & -0.01178 & 0.00000 \\
\hline 20 & 0.9000 & -0.02100 & 0.00000 \\
\hline 21 & 0.8000 & -0.03500 & 0.00000 \\
\hline 22 & 0.7000 & -0.04389 & 0.00000 \\
\hline 23 & 0.6000 & -0.04867 & 0.00000 \\
\hline 24 & 0.5000 & -0.05000 & 0.00000 \\
\hline 25 & 0.4000 & -0.04878 & 0.00000 \\
\hline 26 & 0.3000 & -0.04478 & 0.00000 \\
\hline 27 & 0.2000 & -0.03789 & 0.00000 \\
\hline 28 & 0.1500 & -0.03289 & 0.00000 \\
\hline 29 & 0.1000 & -0.02667 & 0.00000 \\
\hline 30 & 0.0750 & -0.02289 & 0.00000 \\
\hline 31 & 0.0500 & -0.01844 & 0.00000 \\
\hline 32 & 0.0250 & -0.01267 & 0.00000 \\
\hline 33 & 0.0125 & -0.00878 & 0.00000 \\
\hline 34 & 0.0000 & 0.00000 & 0.00000 \\
\hline
\end{tabular}

\subsection{Meshing}

A C-shape domain has been sketched around the airfoil, and the size was $12.5 \mathrm{~m}$ for both the semicircle and the rectangle. The geometry setup is illustrated in figure 5, the airfoil is in the center of the figure.

The Bias factor was set to 150 .

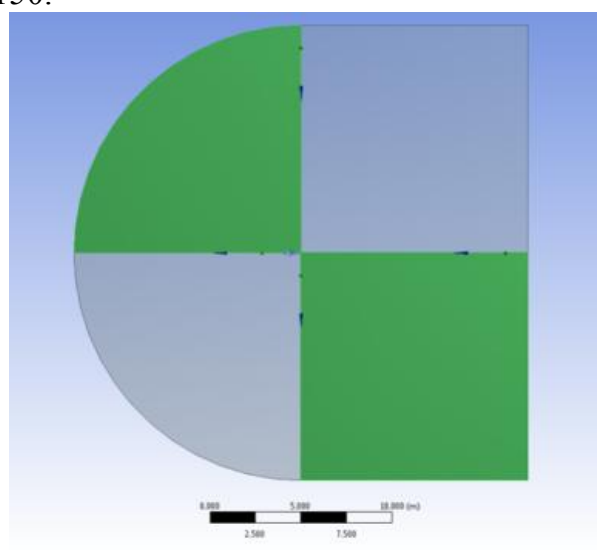

Figure 5. Geometry setup

$$
\% \text { Error }=\frac{\mid \text { Measured Value-Initial Value } \mid}{\text { Initial Value }} \times 100
$$

The Number of divisions was calculated using the above \%Error equation. The angle of attack was set to 3 and run the calculations with three different numbers of divisions which in result showed different numbers of elements and drag forces. Then, the number of division with the lowest \% error was chosen which is 70 as it is showed in Table 2, and the side edge sizing was set to 140, then applied this details to the system for the airfoil. The numbers of setting up were showed in Table 3 . 
Table 2. No. of divisions and \%Error

\begin{tabular}{|l|l|l|l|l|}
\hline Angle of attack & No. Divisions & No. Elements & Drag & \% Error \\
\hline 3 & 50 & 15000 & 50.322226 & \\
\hline 3 & 60 & 21600 & 51.549922 & 2.4396 \\
\hline 3 & 70 & 29400 & 52.446637 & 1.7395 \\
\hline 3 & 80 & 38400 & 54.092778 & 3.138 \\
\hline
\end{tabular}

Table 3. Numbers of setting up

\begin{tabular}{|c|c|}
\hline \multicolumn{2}{|c|}{ NACA 0010-35 } \\
\hline Grid Shape & C-Shape \\
\hline Size & 12.5 \\
\hline No. of Divisions & 70 \\
\hline Side Edge Sizing & 140 \\
\hline Bias Factor & 150 \\
\hline
\end{tabular}

\subsection{Meshing}

C-Mesh was applied to both airfoils in order to guarantee the accuracy of the model by getting more refined mesh over the edge of the trail and the surface of the airfoils. The below figure 6 shows the Cmesh of the simulation:

Figure 6. C-mesh

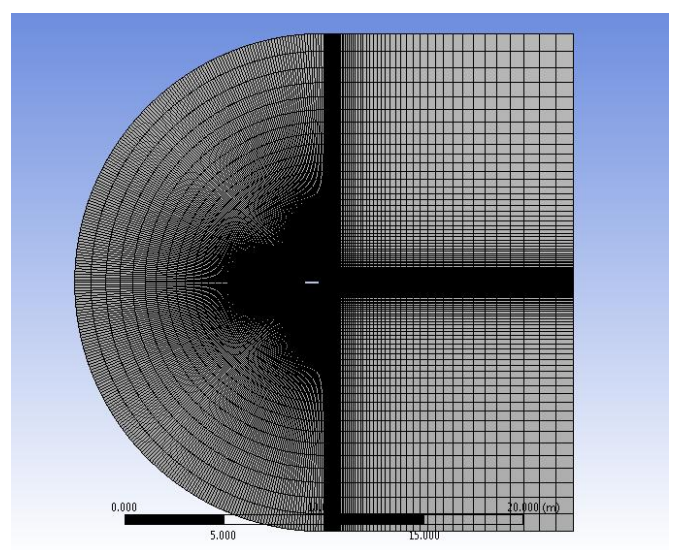

\subsection{Set up the test}

The below settings were used to set up the test:

Model - Solver: Different turbulence models like Spalart- Allmaras, Realizable k- $\varepsilon$ and k- $\omega$ shear street transport had been compared by Eleni et al. [8]. The realizable $\mathrm{k}-\boldsymbol{\varepsilon}$ was used because it is common and popular turbulence model which is mostly used in the industrial applications.

Materials: The Fluid air was used in the category

Boundary conditions zone: The zone condition was selected to be from Inlet.

Monitors: The Residual Convergence which was chosen for the monitors was 1e-6. 
Solution method: Second order upwind was used.

Solution Initialization: The initial values have been computed from the inlet.

Boundary conditions for calculations: The velocity component for $\mathrm{X}$ and $\mathrm{Y}$ directions are calculated as below:

$$
\begin{gathered}
\mathrm{x}=(\text { velocity }) *[\text { Cos }(\text { angle of attack })] \\
\mathrm{y}=(\text { velocity }) *[\text { Sin }(\text { angle of attack })]
\end{gathered}
$$

\section{Other settings:}

- Velocity: 50

- Density: 1

- Pressure: 0

\subsection{Run the calculation}

Number of Iterations : The number of iterations used was 10, 000 to make sure that all the residuals will converge with the increase of the angle of attacks.

\section{Results}

To make the simulations for the airfoil, there were more than 40 running tests have been done with different angle of attacks as the table 4 showed. The results will show the lift and drag coefficients, velocity and pressure behaviors towards the airfoil, and also the effect of airflow over the airfoil.

Table 4. Results

\begin{tabular}{|c|c|c|c|c|c|}
\hline Angle & $\mathrm{x}$-velocity & $\mathrm{y}$-velocity & $\mathrm{Cd}$ & $\mathrm{Cl}$ & Efficiency $(\mathrm{Cl} / \mathrm{Cd})$ \\
\hline-5 & 49.8097349 & -4.5377871 & 0.0355915 & -0.3825206 & -10.7475169 \\
\hline 0 & 50 & 0 & 0.0165975 & $-2.31 \mathrm{E}-04$ & -0.01391463 \\
\hline 2 & 49.9695414 & 1.7449748 & 0.0192779 & $1.57 \mathrm{E}-01$ & 8.133606551 \\
\hline 3 & 49.9314767 & 2.6167978 & 0.0229464 & 0.23148598 & 10.08809686 \\
\hline 4 & 49.878202 & 3.48782368 & 0.0282730 & 0.3023891 & 10.69532443 \\
\hline 5 & 49.8097349 & 4.35778713 & 0.0352934 & 0.36860041 & 10.44388029 \\
\hline 7 & 49.627307 & 6.09346 & 0.0547759 & 0.49069 & 8.958122961 \\
\hline 9 & 49.384417 & 7.8217232 & 0.0810158 & 0.59171372 & 7.303681813 \\
\hline 11 & 49.081359 & 9.5404497 & 0.1118332 & 0.65734985 & 5.877945356 \\
\hline 12 & 48.90738 & 10.395584 & 0.1284684 & 0.67660827 & 5.266726303 \\
\hline 13 & 48.718503 & 11.2475527 & 0.1456626 & 0.68685465 & 4.715379985 \\
\hline 14 & 48.514786 & 12.0960947 & 0.1632566 & 0.68923882 & 4.221812901 \\
\hline 15 & 48.2962913 & 12.9409522 & 0.1812625 & 0.68638258 & 3.786676387 \\
\hline 17 & 47.8152377 & 14.6185852 & 0.2178388 & 0.67328093 & 3.090729788 \\
\hline 18 & 47.5528258 & 15.4508497 & 0.236155 & 0.66704097 & 2.824589655 \\
\hline 19 & 47.275928 & 16.2784077 & 0.2544698 & 0.66275165 & 2.604440659 \\
\hline
\end{tabular}




\subsection{Maximum Lift Coefficient CL (Stalling point) of the Airfoil}

The above table shows that the angle of attack was increased to reach the maximum $\mathrm{Cl}$ (Stalling point). Referring to the table and the figure 7 , it can be seen that as the angle of attack increases, the $\mathrm{Cl}$ increases as well until the angle of attack is set to 14 then the $\mathrm{Cl}$ starts to decrease, so the angle of attack 14 is called the stalling point where it has the maximum $\mathrm{Cl}$ of the airfoil.

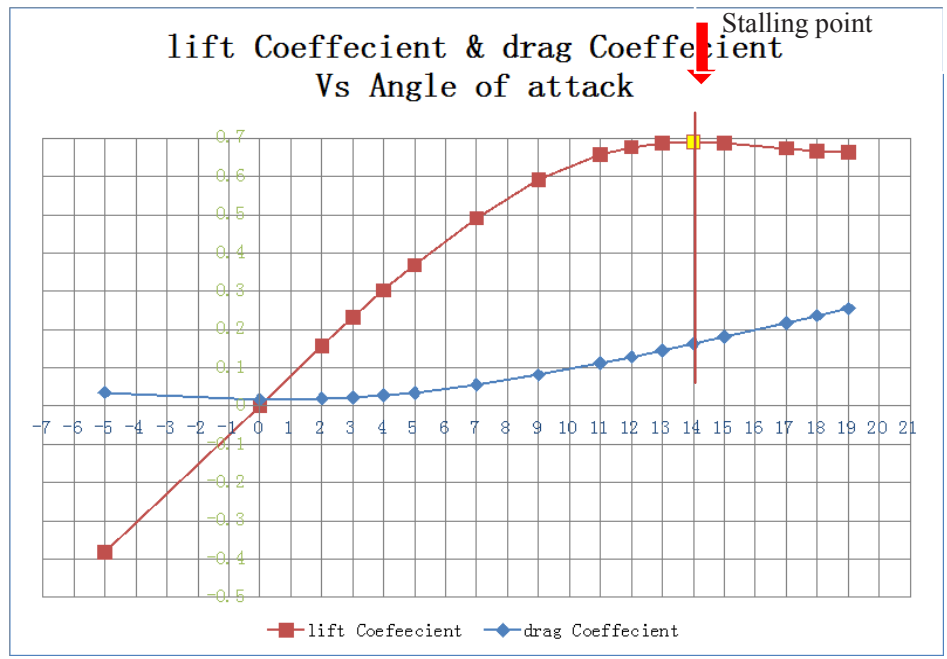

Figure 7. Lift and Drag Coefficients vs Angle of attacks

\subsection{Maximum Efficiency of the Airfoil}

The efficiency is defined as the ratio between $\mathrm{Cl}$ and $\mathrm{Cd}$ at each angle of attack, so referring to the figure 8 , it can be seen that the maximum efficiency is reached at angle of attack (4) for airfoill.

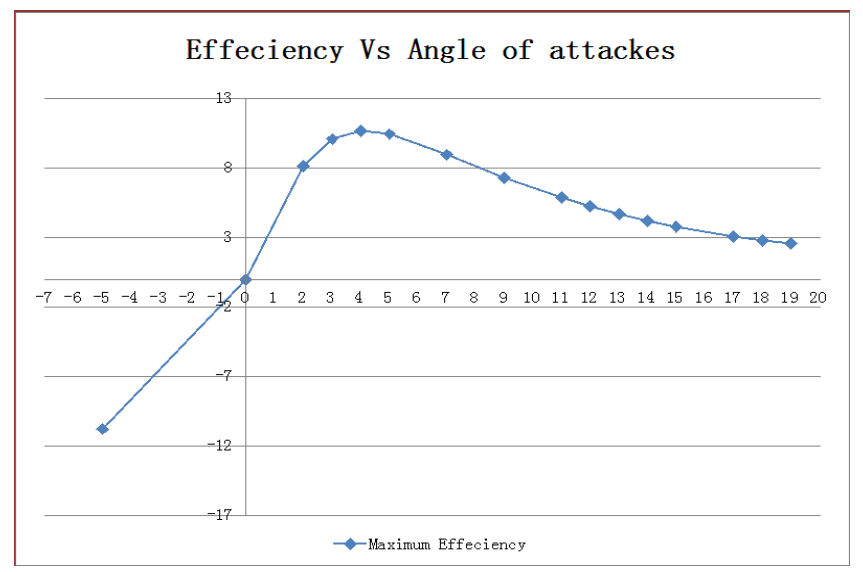

Figure 8. The efficiency vs Angle of attack

\subsection{Contours of static pressure of the airfoil}


It can be seen from the below pictures (figure 9,10,11,12) that when airfoill moves through an airflow and the angle of attack increases, then the pressure will increase gradually and will appear on the bottom surface of airfoil. According to Bernoulli's principle, the upper surface of the airfoil has low pressure while the lower surface has higher pressure, which leads to the flow accelerates on the upper surface and the decrease of the flow velocity at the lower surface.

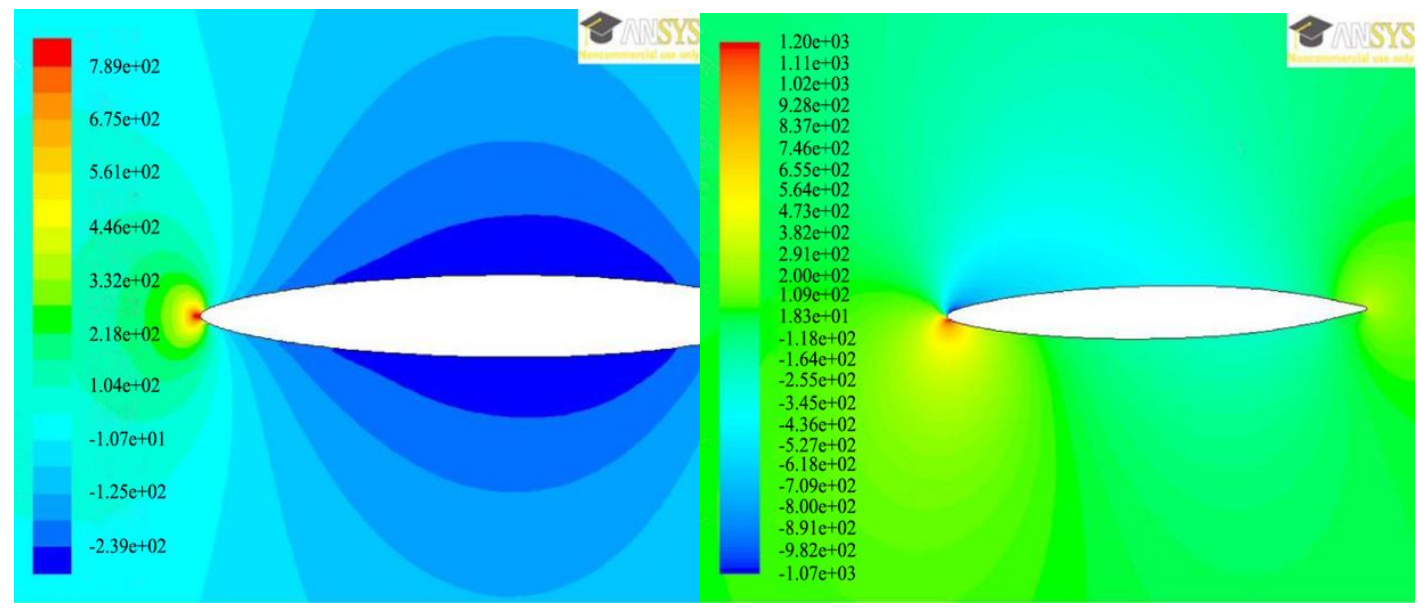

Figure 9. Contours of static pressure $($ Angle $=0)$

Figure 10. Contours of static pressure (Angle $=4)$

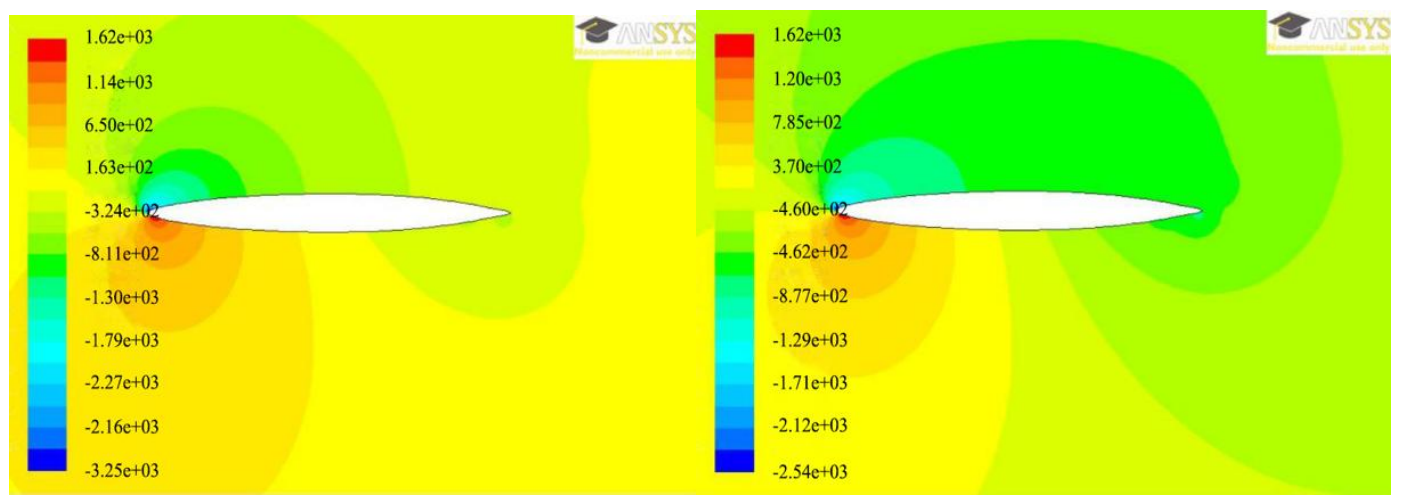

Figure 11. Contours of static pressure $($ Angle=14)

Figure 12. Contours of static pressure $($ Angle $=19)$

\subsection{Contours of velocity magnitude of the airfoil}

It can be seen from the below pictures ( figure 13,14,15,16) that when airfoill moves through an airflow and the angle of attack increases, then the velocity will increase gradually and will appear on the top surface of the airfoil. Also, the air separation is going to be shown clearly when angle of attack increases after the stalling point. 


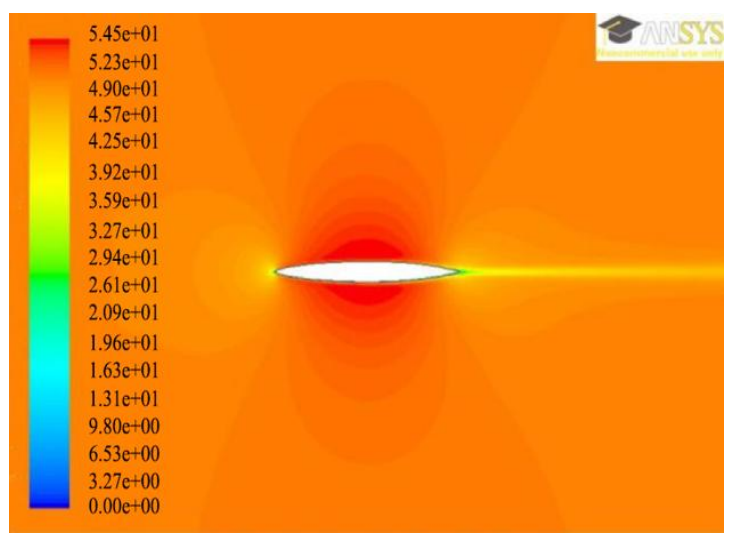

Figure 13. Contours of velocity magnitude (Angle $=0$ )

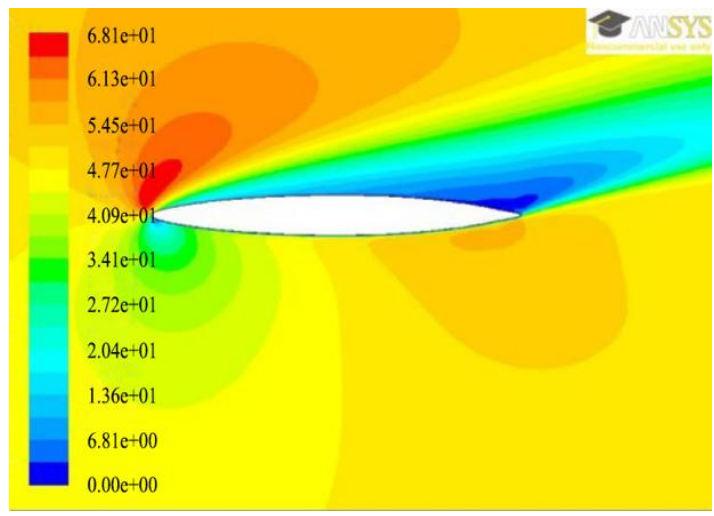

Figure 15. Contours of velocity magnitude (Angle=14)

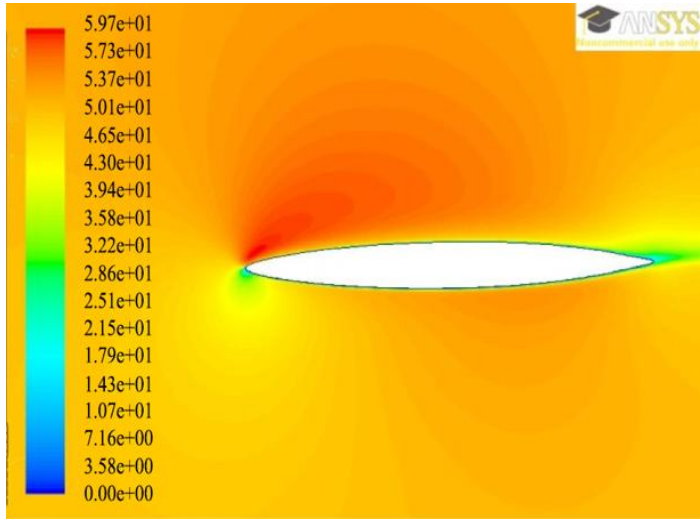

Figure 14. Contours of velocity magnitude (Angle=4)

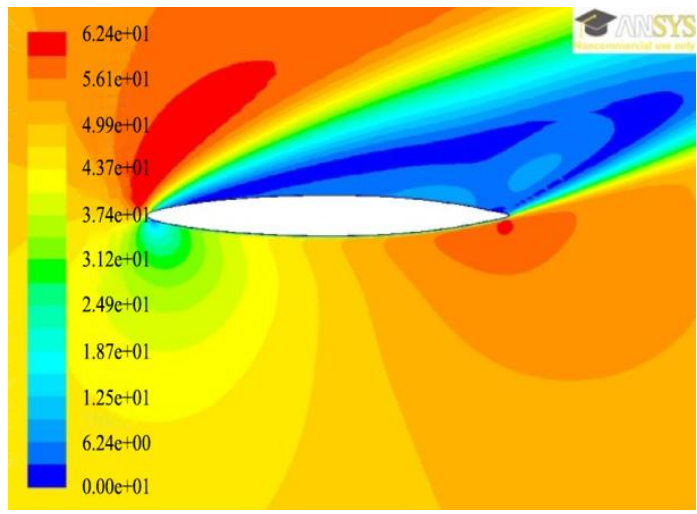

Figure 16. Contours of velocity magnitude (Angle=19)

\subsection{Velocity Stream Function of the Airfoil}

The figure 17,18,19,20 shows that when airfoill moves through an airflow and reaches the Maximum $\mathrm{CL}$, then the air separation will start and a vortex will occur while the angle of attack increases.

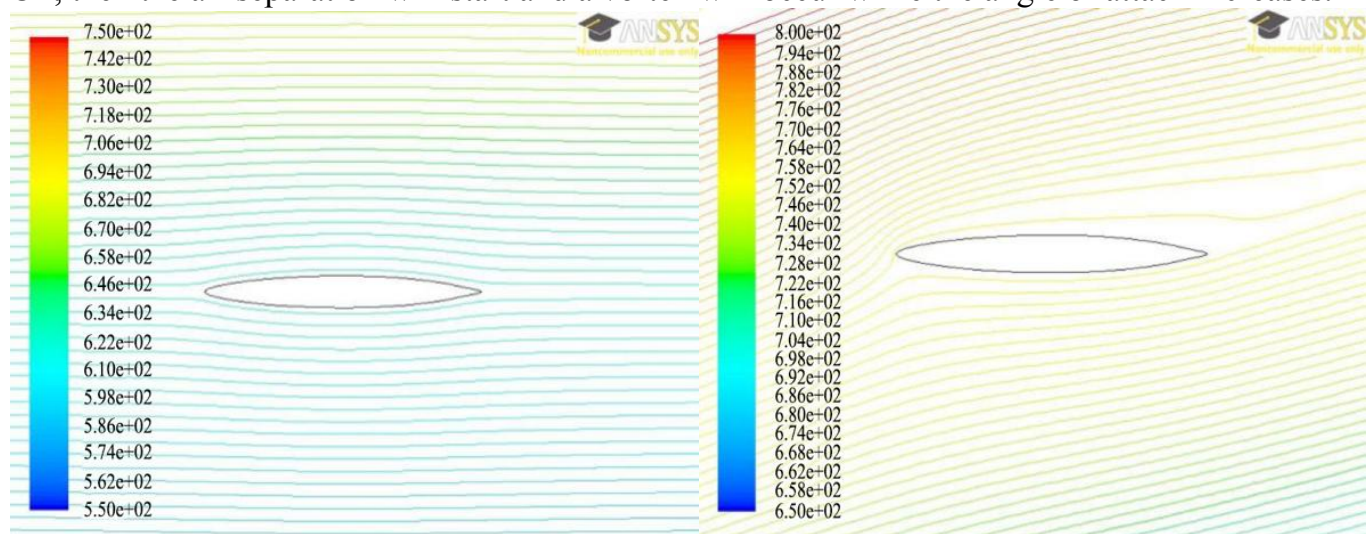

Figure 17. Velocity stream function (Angle $=0)$

Figure 18. Velocity stream function (Angle $=4)$ 


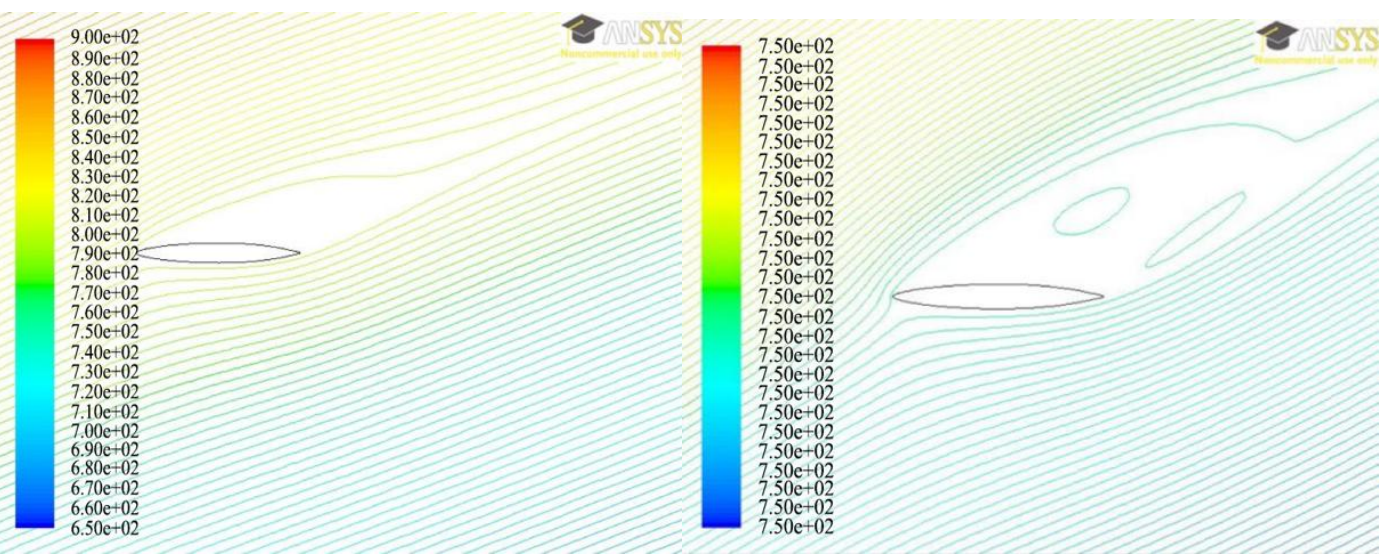

Figure 19. Velocity stream function (Angle $=14$ )

Figure 20. Velocity stream function (Angle=19)

\section{Discussions and Conclusion}

This Symmetrical airfoil was selected and investigated using ANSYS Workbench fluent program Simulation. The aim of this investigation is to reach the maximum Lift Coefficient (stalling point) and the maximum Efficiency for the airfoil through many running calculations with different angle of attacks. By comparing all the results for different angle of attacks, it can be noticed that NACA001035 has the stalling point at the angle of 14. The less stalling point means that the airfoil will have earlier air separation which will help in lifting the airplane. By contrast, when an airfoil reaches the maximum efficiency with angle of attack earlier means that its Maximum Efficiency is more.

\section{Appendix}

The data and geometry of the airfoil NACA0010-35 are downloaded from the UIUC Airfoil Database as follow:

http://m-selig.ae.illinois.edu/ads/coord_database.html\#N

\section{References}

1. T Benson. National Aeronatics and Space Administration. Aerodynamic Forces, retrieved from http://www.grc.nasa.gov/WWW/k-12/airplane/presar.html (n.d.)

2. Cited from Wikipedia: http://en.wikipedia.org/wiki/Lift_coefficient

3. K S Patel, S B Patel, U B Patel, et al. IJER, 3 154-158 (2014)

4. T Benson. National Aeronatics and Space Administration. Aerodynamic Forces, retrieved from http://www.grc.nasa.gov/WWW/k-12/airplane/liftco.html (n.d.)

5. B S Patil, H R Thakare, Procedia Engineering 1271363 - 1369 (2015)

6. I Sahin, A Acir. International Journal of Materials, Mechanics and Manufacturing 31 (2015)

7. S S Bhat, R N Govardhan. Journal of Fluids and Structures 41 166-174 (2013)

8. D C Eleni, T I Athanasios, M P Dionissios.JMER 4 (3) 100-111(2012) 